\title{
Modeling Learning and Strategy Formation as Phase Transitions in Cortical Networks
}

\author{
Robert Kozma, Yury Sokolov, Marko Puljic \\ Department of Mathematics \\ University of Memphis \\ Memphis, TN, 38152, USA \\ Email:\{rkozma, ysokolov\}@memphis.edu
}

\author{
Sanquing $\mathrm{Hu}$ \\ College of Computer Science \\ Hangzhou Dianzi University \\ Hangzhou, China \\ Email: sqhu@hdu.edu.cn
}

\author{
Miklós Ruszinkó \\ Alféd Rényi Institute of Mathematics \\ Hungarian Academy of Sciences \\ Budapest, 1053, Hungary \\ Email: ruszinko.miklos@ renyi.mta.hu
}

\begin{abstract}
Learning in the mammalian brain is commonly modeled through changing synaptic connections in cortical networks. Dynamical brain models indicate that learning leads to the formation of limit cycle oscillations across cortical areas and that the oscillatory regimes re-emerge when the learnt input is presented to the system. In this work, learning is modeled using a graph-theoretical model, which captures salient characteristics of the learning process. We introduce a random graph that combines a torus with lattice edges and additional random edges, which have power law length distribution. On this graph, we consider bootstrap percolation with excitatory and inhibitory vertices. Theoretical and numerical studies indicate the presence of various dynamical regimes on these graphs. Here, the transitions between fixed-point and limit cycle attractors are analyzed. We link this transition to changes in cortical networks during category learning, which have been observed in animal experiments using electro-cortiograph (ECoG) arrays over sensory cortices. We discuss how learning leads to categorization and strategy formation, and how the theoretical modeling results can be used for designing learning and adaptation in computationally aware intelligent machines.
\end{abstract}

\section{INTRODUCTION}

A fundamental challenge of decision making is to identify a scenario describing the actual situation of a biological or artificial system at a given instance. Once a suitable scenario is identified out of many possible scenarios, a corresponding strategy can be selected to achieve the system's goal in the given context [9]. If the system's internal conditions or the external environment change, the scenario changes as well, which requires a change in strategy that corresponds the best way to the new situation [3]. Animals have the capacity of rapidly changing their strategy and the corresponding actions if needed, e.g., changing from a quiet grazing behavior to the escape mode if a threat is perceived. However, strategy change is still a hardly understood area of cognitive science. Behavioral and physiological data indicate the presence of frequent, sudden changes in strategies, but a consistent theory of the underlying neurodynamic processes is still missing [14].

Recent progress in studying biological learning processes may provide important insights into the mechanism of strategy change. Most of the current physiological and theoretical frameworks of learning focus on incremental adaptation mechanisms, as exemplified by the reinforcement learning framework, while the discontinuous nature of behavioral development observed in strategy change is not well understood. Here, we summarize experimental evidence of sudden changes during the learning process. Moments of sudden insight and deep understanding are not unfamiliar in our everyday learning practice. This is colloquially referred to as the "Aha" moment when you suddenly exclaim, after minutes or hours of trying to make sense of some problem: "Aha, of course, now I do understand!"

We employ a neurodynamic theory based on the hierarchy of Freeman K sets, including K0, KI, KII, KIII, and KIV sets corresponding to increasing complexity of structure, dynamics, and function [2], [10], [11]. K0 sets describe the basic openloop nonlinear unit converting pulse density to wave density, as the basic building block of neurodynamics. KI is a population of excitatory or inhibitory $\mathrm{K} 0$ units, which can generate nonzero fixed point dynamics for a sufficient level of mutual interaction. KII sets consist of interacting excitatory and inhibitory KI sets producing limit cycle oscillations at specific frequency bands [16]. Higher level K models include KIII and KIV sets, which describe the intentional action-perception cycle and awareness experience [10].

In this paper, we introduce a graph theoretical model featuring phase transition behavior, to interpret learning effects observed in experiments. The present work focuses on KI and KII sets, the stability properties of which has been studied, e.g., in [16]. We develop a two-dimensional graph to model the cortical neuropil with short and long edges describing the combination of short dendritic connections and long axons. The long edges have power law length distributions, leading to drastic reduction of the diameter of the brain graph, i.e., with diameter of the order of $\log (n)$ in an $n \times n$ lattice [7]. Our model incorporates excitatory-inhibitory populations as given by Freeman KII sets, and we define an activation process over the graph describing propagating volleys of pulse densities across the cortex. We derive rigorous mathematical conditions for the emergence of self-sustained background activity in excitatory neural populations as phase transitions, depending on the importance of long edges. Phase transitions from non-zero fixed point to oscillatory behavior are characterized. Learning 
has been previously addressed in $\mathrm{K}$ models using Hebbian rule [11]. Learning in this work is described through changes in the update rule describing mutual activation of connected nodes and changes in long-range connectivity. Learning leads to sudden onset of large connected components near criticality, interpreted as the formation of Hebbian cell assemblies. We conclude with discussions concerning the use of the introduced results for the development of artificially intelligent systems, which show emergent properties of awareness experience while exploring the environment.

\section{EXPERIMENTAL}

Learning in the mammalian brain is commonly modeled via changing synaptic connections in cortical networks. According to Freeman's dynamic brain model [3], synaptic learning modifies the attractor landscape by forming localized attractor basins manifested through amplitude modulation (AM) patterns of spatially distributed cortical activity with narrow-band oscillations. Once an input category is stored in the dynamic attractor landscape, that input evokes a specific AM pattern in a narrow frequency band every time it is presented to the system. The re-emergence of the AM pattern indicates successful recall of the learnt category through the collapse of the complex dynamics to a localized basin. The AM pattern manifests the meaning of the input stimulus as perceived by the animal and it leads to the selection of a corresponding action [4].

In a collaborative project, we exploit the experimental and theoretical accessibility of a particular rodent learning model, to analyze the neuronal mechanisms of behavioral strategy change. Experiments are conducted at LIN, Germany using multi-electrode arrays measuring surface potentials over the auditory cortex (4x5 array) and local field potentials in the ventral striatum of Mongolian gerbils trained using wellestablished Go/NoGo discrimination paradigm. The gerbil learns to move from one side of the cage to the other when a rising tone is heard. This is the Go signal, and the gerbil receives a negative reinforcement signal (a small electric shock through the bottom grid of the box) if it does not move in response to the Go signal. On the other hand, the gerbil is supposed remain in its present position in the case of a falling tone (No-Go signal). The experiments document sudden transitions in the behavior as learning progresses, i.e., the gerbil is in a naive state (no learning) in the first part of the experiments without significant improvement in its learning performance. However, at a given time point it suddenly exhibits significantly improved performance, and maintains this good performance there after. This is the "aha" moment of gerbil, when it learns how to avoid the penalty signal. For details of the experimental paradigm, see, [14], [15], [6]. Our analysis at present focuses on data obtained over the auditory cortex (AC) with Go or No-Go conditions during a training sequence of 4 to 6 days for any given gerbil. Gerbils typically perform poorly in the first few days, however, they exhibit sudden improvement in their performance afterwards. For some "smart" gerbils, the learning is completed during

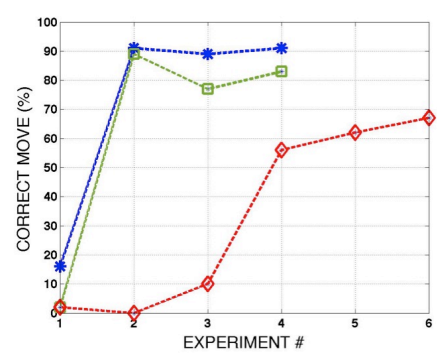

Fig. 1. Learning curves observed in the gerbil experiments. The first two (smart) gerbils learn the task in Day 1, and demonstrate good performance the remaining trials; see the curves marked by stars and squares (blue and green). Gerbil 3 needs three days to learn the same task, see diamonds (red) and even then its performance lags the other two (smart) gerbils.

the first day, and they produce the required move correctly from day two onwards; see Fig. 1, curves marked by with stars and squares. For other gerbils it may take two or even three days to learn the task, Fig. 1, curve with diamond. Once they learn to categorize the auditory signal, their good categorization and action selection performance is maintained for the rest of the trials. Our analysis confirms that measurements produce classifiable amplitude modulation (AM) patterns in the ECoG array over the auditory cortex in the gamma band, in agreement with expectations [6].

Using a generalized Granger causality metric (NC) [5], [12], we document the formation of enhanced connectivity between certain electrode locations in the auditory cortex as learning progresses, reaching a plateau after day 2 or 3 [6]. Such behavior supports the hypothesis about the formation of Hebbian cell assemblies (HCA) during reinforcement learning, leading to a phase transition when a critical connectivity density pattern is reached. This is the motivation of the graph theoretical model in the present work, when we show how structural changes in a critical network model can produce dynamic behaviors (narrow-band oscillations) as the result of the learning process.

\section{RANDOM GRAPH MODEL}

\section{A. Definition of the random graph over the lattice}

Since the early 2000's, random graph theory has been used successfully to describe the interaction between the structure and dynamics of cortical networks [8], [1]. Based on those foundations, recently, we introduced a random graph model $G_{\mathbb{Z}_{N}^{2}, p_{d}}$ that consists of a square grid with periodic boundary conditions, torus, and additional random edges in [7]. The probability of random edge between a pair of vertices is inversely proportional to the graph distance between the vertices defined on the torus. The random edges are called long and the edges of the torus short. The definition of the model captures an important property of cortical networks, that is, it is more likely for a neuron to have connections to nearby neurons than to neurons which are far from the given one. 

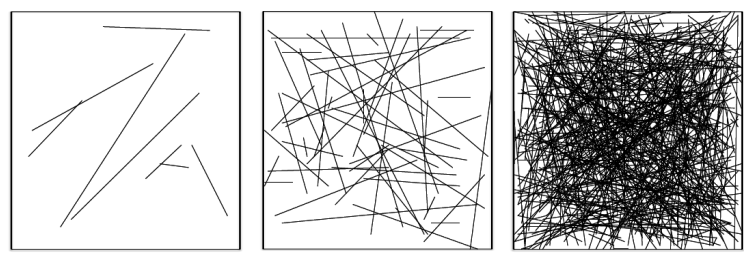

Fig. 2. Illustration of the additional long edges over the square lattice. The three plots correspond to 8, 53, and 256 randomly added long edges (from left to right), respectively; the original edges of the $64 \times 64$ lattice are not shown.

The model is defined as follows. The set of vertices of the random graph $G_{\mathbb{Z}_{N}^{2}, p_{d}}$ is given by the vertex set of $\mathbb{Z}_{N}^{2}$, where $\mathbb{Z}_{N}^{2}$ is a torus on $N^{2}$ vertices. All the edges of the torus $\mathbb{Z}_{N}^{2}$ are presented in $G_{\mathbb{Z}_{N}^{2}, p_{d}}$. The random edges of $G_{\mathbb{Z}_{N}^{2}, p_{d}}$ are distant-dependent, i.e., the probability that an arbitrary pair of vertices, $u, v$, that are at graph distance $d$ apart of each other, is given by

$$
p_{d}=\mathbb{P}\left((u, v) \in E\left(G_{\mathbb{Z}_{N}^{2}, p_{d}}\right) \mid \operatorname{dist}(u, v)=d\right)=\frac{c}{N d^{\alpha}},
$$

where $d>1, c$ is a positive constant, and $\alpha$ is the power exponent of long edge length distribution. In this work, we use $\alpha=1$, for simplicity. It is assumed that there are no multiple edges between the vertices. Examples of graphs with additional edges are shown in Fig. 2; there are 8, 53, and 256 edges added in the plots from left to right, respectively.

\section{B. Properties of the random graph model}

Here we summarize some properties of the random graph; detailed exposition is given in [7]. First we note that the addition of random edges drastically reduces the diameter $(D)$ of the graph. Clearly, the square lattice has diameter $N$. We show that the diameter decreases from linear in the number of vertices to logarithmic, when long edges are added, i.e., the following holds:

$$
D\left(G_{\mathbb{Z}_{N}^{2}, p_{d}}\right) \sim(\log N)
$$

This graph property is important for cortical networks since the addition of long edges may lead to faster propagation of activity over the network. For example, consider $10^{10}$ units arranged over a square lattice $N \times N$, where $N=10^{5}$. The diameter of the regular torus on this lattice (without long edges) is $d \sim 10^{5}$. However, adding some random edges, reduces the diameter to $D \sim \log \left(10^{5}\right) \sim 5$. This example corresponds to the case of small mammalian, such as gerbils, and it shows the importance of long edges in defining the brain network structure.

From the above fact it follows that addition of random edges changes drastically the structure of the torus. Moreover, the random graph is no longer a regular graph as it is the torus. To see this let us describe the degree probability distribution. Without long edges, this distribution is uniform, i.e., all vertices have 4 edges to their neighbors in a square lattice. When adding long edges randomly, it is possible to approximate the degree distribution with respect to long edges by a Poisson probability distribution. Let $W$ be the random variable describing the degree of a vertex with respect to long edges only. Then the degree distribution of the vertex including long and short edges is $W+4$. We have the following property for the probability distribution of long edges; for a proof of the lemma see [7]. The probability that a vertex has degree $k$ considering the long edges is given by

$\mathbb{P}(W=k)=\sum_{k_{2}+\ldots+k_{N}=k} \prod_{i=2}^{N}\left(\begin{array}{c}\left|\Lambda_{i}\right| \\ k_{i}\end{array}\right)\left(\frac{c}{N i}\right)^{k_{i}}\left(1-\frac{c}{N i}\right)^{\left|\Lambda_{i}\right|-k_{i}}$

The total variation distance $d_{T V}$ satisfies

$$
d_{T V}=\frac{1}{2} \sum_{j \geq 0}|\mathbb{P}(W=j)-\mathbb{P}(Y=j)|=O(1 / N),
$$

where $W$ is the degree distribution of our random graph, while $Y$ is a random variable with Poisson distribution $\operatorname{Po}(\lambda)$, with $\lambda=4 c \ln 2$. Thus the degree distribution of our random graph approaches Poissonian law better and better as the size $N$ increases. It is natural to expect that as $\lambda$ increases the number of additional random edges growth, which is rigorously proven in [7].

\section{Bootstrap percolation on $G_{\mathbb{Z}_{N}^{2}, p_{d}}$}

The spread of activity in the brain can be described by a simplified mathematical model - bootstrap percolation (BP). We state here a generalized definition of bootstrap percolation that consists of two types of vertices, which correspond to excitatory and inhibitory units. Bootstrap percolation with one type of vertices (excitatory or inhibitory) can be viewed then as a special case. Each vertex of the graph is described by two random variables, its type and state. The types of vertices are excitatory (1) or inhibitory (2). We define the type as a Bernoulli random variable $B e(\omega)$, which is selected at the start of the process and remains unchanged afterwards. In contrast, state of a vertex may change during the process as follow. At each time step a vertex is either active or inactive. For each vertex $v$ of the graph we assign a binary function $\chi_{v}(t)$ which describes the activity state of the vertex at time $t$. A vertex is said to be active if $\chi_{v}(t)=1$, otherwise it is inactive and $\chi_{v}(t)=0$. For each vertex $v$, the potential function $\chi_{v}(0)$ at the beginning is a Bernoulli random variable $\operatorname{Be}(p)$.

Let $A(t)=\left\{v \in V\left(G_{\mathbb{Z}_{N}^{2}, p_{d}}\right) \quad \chi_{v}(t)=\right.$ $1 \& v$ is of type $i\}, i \in\{E, I\}$ denote the set of all active vertices at time $t$, while $A_{E}(t)$ and $A_{I}(t)$ are the set of active vertices of type $E$ and $I$ at time $t$, respectively. Thus, $A(t)=A_{E}(t) \cup A_{I}(t) . A(0)$ consists of all vertices that are active at the beginning. Each vertex may change its activity based on the states of its neighbors. For a vertex $v$ which is of type $E$, the evolution rule is given by

$$
\chi_{v}(t+1)=\mathbb{1}\left(\sum_{u \in N^{E}(v)} \chi_{u}(t)-\sum_{u \in N^{I}(v)} \chi_{u}(t) \geq k\right)
$$


where $N^{E}(v)$ and $N^{I}(v)$ denote the subset of vertices in the closed neighborhood of vertex $v$, which are of $E$ and $I$ type, respectively; and $\mathbb{1}$ is the indicator function. For a vertex $v$ of type $I$, the following rule holds

$$
\begin{array}{r}
\chi_{v}(t+1)=\mathbb{1}\left(\sum_{u \in N^{E}(v)} \chi_{u}(t)+\sum_{u \in N^{I}(v)} \chi_{u}(t) \geq k\right)= \\
\mathbb{1}\left(\sum_{u \in N(v)} \chi_{u}(t) \geq k\right), \quad(5)
\end{array}
$$

where $N(v)=N^{E}(v) \cup N^{I}(v)$ is the closed neighborhood of vertex $v$. The rules try to capture the significant difference and importance of each time in neurodynamics.
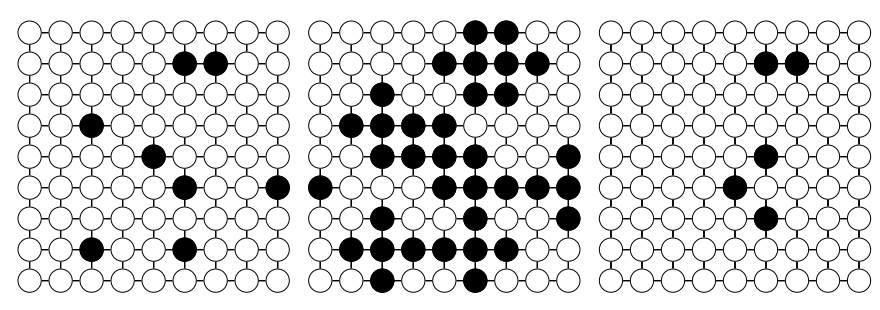

Fig. 3. Illustration of the update rule over the square lattice; black dots are active sties, empty circles indicate inactive sites. The leftmost subplot shows the initial activation at $t=0$; the center plot is the activation at the next time instant $t=1$ for $k=1$; the right plot is the case $t=1$ and $k=2$.

The bootstrap percolation rule is illustrated in Fig. 3 in the case of $k=1$ and $k=2$. For simplicity, only excitatory nodes are considered, and there is no long edges added $(\lambda=0)$. Initializing a few vertices at $t=0$ marked by black circles in the left plot, all the neighbors of the initial active nodes are active in the case of $k=1$ (center plot). For $k=2$, however, only one nodes remain action at time $t=1$, i.e., the one which had at least 2 active neighbors in the previous step. The rigorous mathematical analysis of the percolation process in the $2 \mathrm{D}$ random graph with long additional edges is a difficult mathematical problem, see, e.g. [13].

\section{MEAN-FIELD APPROXIMATION IN BOOTSTRAP PERCOLATION}

\section{A. The case of one type of (excitatory) vertices}

In mean-field (MF) approximation it is assumed that the states and degrees are well-mixed, moreover, different vertices are regarded as independent. Under these assumptions $\rho_{t}$ can be well-approximated by its mean, where $\rho_{t}=A(t) / N^{2}$, and $N^{2}$ is the size of the torus. This can be stated more precisely as follows. Let $\operatorname{Bin}(n, p)$ be a binomial random variable. Then the density $\rho_{t}$ in the mean-field model satisfies the following stochastic recursion.

Lemma 1: For the mean-field approximation on graph $G_{\mathbb{Z}_{N}^{2}, p_{d}}$ with $N^{2}$ nodes, $\rho_{t}$ is a Markov process given by

$$
N^{2} \rho_{t+1}=\operatorname{Bin}\left(N^{2} \rho_{t}, f^{+}\left(\rho_{t}\right)\right)+\operatorname{Bin}\left(N^{2}\left(1-\rho_{t}\right), f^{-}\left(\rho_{t}\right)\right),
$$

where

$f^{+}(x)=\sum_{n=4}^{N^{2}-1} \mathbb{P}\left(\operatorname{deg}(v)=n_{o}\right) \sum_{i=k}^{n+1}\left(\begin{array}{c}n \\ i-1\end{array}\right) x^{i-1}(1-x)^{n-i+1}$

$f^{-}(x)=\sum_{n=4}^{N^{2}-1} \mathbb{P}\left(\operatorname{deg}(v)=n_{o}\right) \sum_{i=k}^{n}\left(\begin{array}{l}n \\ i\end{array}\right) x^{i}(1-x)^{n-i}$,

where $n_{o}=n-4$ is the degree based on random edges only. For a given $\rho_{t}, \rho_{t+1}$ has mean $f\left(\rho_{t}\right)$ and variance $g\left(\rho_{t}\right) / N^{2}$ where

$$
\begin{aligned}
& f(x)=x f^{+}(x)+(1-x) f^{-}(x) \\
& g(x)=x f^{+}(x)\left(1-f^{+}(x)\right)+(1-x) f^{-}(x)\left(1-f^{+}(x)\right)
\end{aligned}
$$

The rigorous proof of the existence of phase transition in the random graph is proven in the main theorem of [7]. Based on the analysis of the above Markov process, it can be summarized as follows:

Theorem 1: In the mean-field approximation of the bootstrap percolation wtih one type of vertices on the random graph $G_{\mathbb{Z}_{N}^{2}, p_{d}}$ there exists a critical probability $p_{c}$ such that for a fixed $p$, with high probability for large $N$, all vertices will eventually be active if $p>p_{c}$, while all vertices will eventually be inactive for $p<p_{c}$. The value of $p_{c}$ is given as the function of $k$ and $\lambda$ as follows:

(i) For $k=0$ and any $\lambda, p_{c}=0$ and all vertices will become active in one step for any $p$.

(ii) For $k=1$ and any $\lambda, p_{c}=0$, i.e., for any fixed $p>$ 0 , all vertices will eventually become active with high probability.

(iii) For $k=2$ and any $\lambda, p_{c}=x_{2}(\lambda)$, where $x_{2}(\lambda) \in(0,1)$ is a nontrivial solution to $x=\bar{f}_{2}(x)$.

(iv) For $k=3$ and any $\lambda, p_{c}=x_{3}(\lambda)$, where $x_{3}(\lambda) \in(0,1)$ is a nontrivial solution to $x=\bar{f}_{3}(x)$.

One of the corollaries of this theorem is that critical probability $p_{c}$ in cases $k=2,3$ decays with respect to $\lambda$. This means that in the presence of one type of vertices activation spreads faster as the number of edges increases. In particular, when $k=2$ we have $p_{c} \leq x_{2}(0)=0.5$, and when $k=3$ the critical probability is bounded by $p_{c} \leq x_{3}(0)=0.131$. Detailed evaluations indicate high value of $p_{c}$ for $\lambda$ values up to about 100 , and a drop of the critical probability in the range of $\lambda \in\left[10^{2}, 10^{3}\right]$. For $\lambda>10^{3}$, the critical probability diminishes for both $k$ values.

\section{B. Mean-field approximation of BP with two types of (excita- tory and inhibitory) vertices}

In the case when there are two types of vertices we need to take into account the evolution of two density functions that correspond to active nodes of each type. Let

$$
\rho_{t}^{(1)}=\frac{\left|A_{1}(t)\right|}{\omega N^{2}}
$$


and

$$
\rho_{t}^{(2)}=\frac{\left|A_{2}(t)\right|}{(1-\omega) N^{2}}
$$

be the densities of the first and second types, correspondingly, where $A_{i}(t)$ is the number of active vertices of type $i, i=1,2$, at time $t$. Then, in particular, the density of all active nodes is given by

$$
\rho_{t}=\omega \rho_{t}^{(1)}+(1-\omega) \rho_{t}^{(2)}=\frac{\left|A_{1}(t)\right|+\left|A_{1}(t)\right|}{N^{2}} .
$$

Similarly as for the BP with one type of vertex, it is possible to derive mean-field approximation of BP with two types; here we summarize the results [13].

Lemma 2: For the mean-field approximation on graph $G_{\mathbb{Z}_{N}^{2}, p_{d}}$ with $N^{2}$ nodes, which are of the two types and a vertex is of the first type with probability $w, \rho_{t}^{(i)}, i=1,2$, are defined by

$$
\begin{array}{r}
w N^{2} \rho_{t+1}^{(1)}=\operatorname{Bin}\left(w N^{2} \rho_{t}^{(1)}, f_{1}^{+}\left(\rho_{t}^{(1)}, \rho_{t}^{(2)}\right)\right) \\
\quad+\operatorname{Bin}\left(w N^{2}\left(1-\rho_{t}^{(1)}\right), f_{1}^{-}\left(\rho_{t}^{(1)}, \rho_{t}^{(2)}\right)\right),
\end{array}
$$

$$
\begin{array}{r}
(1-w) N^{2} \rho_{t+1}^{(2)}=\operatorname{Bin}\left((1-w) N^{2} \rho_{t}^{(2)}, f_{2}^{+}\left(\rho_{t}^{(1)}, \rho_{t}^{(2)}\right)\right) \\
+\operatorname{Bin}\left((1-w) N^{2}\left(1-\rho_{t}^{(2)}\right), f_{2}^{-}\left(\rho_{t}^{(1)}, \rho_{t}^{(2)}\right)\right),
\end{array}
$$

Moreover, $\rho_{t+1}^{(i)}$ is given by a distribution with mean $f_{i}\left(\rho_{t}^{(1)}, \rho_{t}^{(2)}\right)$ and variance $g_{i}\left(\rho_{t}^{(1)}, \rho_{t}^{(2)}\right) / N^{2}, i=1,2$, where

$$
\begin{gathered}
f_{1}(x, y)=x f_{1}^{+}(x, y)+(1-x) f_{1}^{-}(x, y) \\
f_{2}(x, y)=y f_{2}^{+}(x, y)+(1-y) f_{2}^{-}(x, y) \\
g_{1}(x, y)=x f_{1}^{+}(x, y)\left(1-f_{1}^{+}(x, y)\right)+ \\
(1-x) f_{1}^{-}(x, y)\left(1-f_{1}^{+}(x, y)\right) \\
g_{2}(x, y)=y f_{2}^{+}(x, y)\left(1-f_{2}^{+}(x, y)\right)+ \\
(1-y) f_{2}^{-}(x, y)\left(1-f_{2}^{+}(x, y)\right)
\end{gathered}
$$

Functions $f_{j}^{ \pm}$, for $j=1,2$ are defined as follows

$$
\begin{gathered}
f_{1}^{+}(x, y, \omega)=\sum_{n=4}^{N^{2}-1} \mathbb{P}(\operatorname{deg}(v)=n-4) \sum_{i=1}^{n+1} p_{i}^{1+}(x, y, \omega) \\
\left(\begin{array}{c}
n \\
i-1
\end{array}\right)(\omega x+(1-\omega) y)^{i-1}(1-\omega x-(1-\omega) y)^{n-i+1} \\
f_{1}^{-}(x, y, \omega)=\sum_{n=4}^{N^{2}-1} \mathbb{P}(\operatorname{deg}(v)=n-4) \sum_{i=0}^{n} p_{i}^{1-}(x, y, \omega) \\
\left(\begin{array}{c}
n \\
i
\end{array}\right)(\omega x+(1-\omega) y)^{i}(1-\omega x-(1-\omega) y)^{n-i}(20) \\
f_{2}^{+}(x, y, \omega)=\sum_{n=4}^{N^{2}-1} \mathbb{P}(\operatorname{deg}(v)=n-4) \sum_{i=1}^{n+1} p_{i}^{2+} \\
\left(\begin{array}{c}
n \\
i-1
\end{array}\right)(\omega x+(1-\omega) y)^{i-1}(1-\omega x-(1-\omega) y)^{n-i+1}
\end{gathered}
$$


a wide range of $\lambda, \omega$, and $p$ values. The type of dynamics for a fixed parameter triple has been identified after omitting the initial transients. Since the type of a vertex is determined at the beginning uniformly at random, thus $\omega$ defines the overall ratio of excitatory and inhibitory nodes, which is unchanged during the process. It is observed that limit cycle behavior belongs to excitatory to inhibitory ratio of around $4 / 1$.

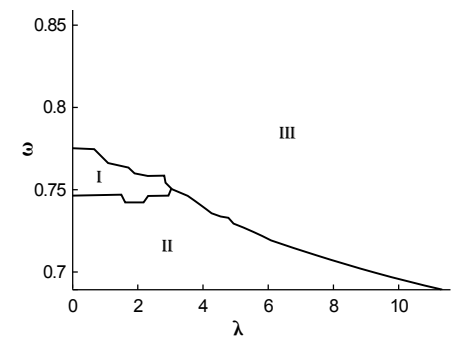

Fig. 4. Bifurcation diagram of BP with two types, where $k_{1}=2$ and $k_{2}=3$. Limit cycle dynamics exists in region I, processes of both types eventually die out in II, and non-zero fixed point dynamics exist in region III.

\section{DISCUSSION OF GERBIL LEARNING BASED ON RESULTS WITH THE GRAPH MODEL}

In this section we analyze the potential role several key model parameters play in interpreting the learning dynamics of the cortical tissue. Namely, we study the following parameters: the significance of long edges $(\lambda)$ that create small-world phenomena; the ratio of the number of excitatory versus inhibitory nodes $(\omega)$ related to the onset of narrow band oscillations; and threshold values of the update rule $\left(k_{1}\right.$ and $k_{2}$ ), which define the sensitivity of the node to changes in its neighborhood. For the purpose of the present analysis, we focus on possible changes in $k_{1}$ and $k_{2}$ due to learning, as well as the evolution of the non-local connectivity through $\lambda$, while we do not discuss possible impact due to changes in $\omega$.

Previously, we conducted causality analysis of experimental data obtained during category learning in gerbils, and we documented the formation of functional connections in the auditory cortex. The applied signal processing approach has been introduced previously in the literature as "New Causality (NC)" [5]. NC is a generalization of Granger causality (GC), which evaluates the role one time series play in predicting another time series; for details see [6].

We collected EEG channel pairs over a $4 \times 5$ grid, and searched for pairs with different bidirectional causality flows for the two types of stimuli, i.e., for the Go and No-Go signals. We divided the total duration of the experiments in two parts. In Part I the animal does not react well to the Go signal; this is naive state of the animals. Part I is Day 1 for the two smart gerbils in Fig. 1 (stars and circles), and it takes Days 1, 2, and 3 for the third gerbil (diamonds). Part II corresponds to well-trained behavior, that consists of Days 2, 3, and 4 for the smart gerbils, and Days 4, 5, and 6 for the third gerbil. We counted the electrode pairs which had causality relationship in the sense of NC.
The results are summarized in Table I. There are causal pairs both in the naive stage (Type I) and trained stage (Type II), but the number of causal pairs is larger in the case of trained animal (Type II). This conclusion has been tested and verified with a number of other animals, in total 7 gerbils have been analyzed in [6]. A systematic statistical test (based on paired t-test) shows that the hypothesis that the number of pairs is larger in Type II that in Type I is valid with high confidence $(\mathrm{p}=0.01)$.

TABLE I

Number of CAUSAlity Pairs For 3 Gerbils

\begin{tabular}{|c|c|c|c|}
\hline Type & Gerbil 1 & Gerbil 2 & Gerbil 3 \\
\hline I (Naive) & 11 & 3 & 8 \\
\hline II (Trained) & 50 & 13 & 49 \\
\hline
\end{tabular}

This is an important result showing that we can detect neural correlates of reinforcement learning in the gerbil's cortex using NC measures. The difference between the behavior of the naive and trained animal has now a experimentally observable neural measure through the formation of connections (causal links) between certain cortical regions as the result of learning. These links and regions may be the manifestations of the formation of Hebbian Cell assemblies (HCA) [3]. Until now there have been very few direct evidences for HCAs; our results in fact may provide such evidence. Based on Freeman neurodynamics, we know that learning is closely related to the onset of narrow band oscillations in the cortex as a consequence of sustained reinforcement signal. This observation provides an important information for our modeling studies.

Our graph model can provide theoretical insights to interpret the previously described experimental results. Our model exhibit phase transitions between fixed point and limit cycle dynamics when certain control parameters of the model vary. Here let us us focus on the significance of long edges $(\lambda)$ and threshold values of the update rule $\left(k_{1}\right.$ and $\left.k_{2}\right)$. For example, in the case of $k_{1}=k_{2}=3$ our model show fixed point behavior, which can change to oscillatory behavior if $k_{1}$ is decreased to 2 . Decreasing $k_{1}$ can be interpreted as the reduction of the dynamic threshold in the interaction between the cortical locations due to learning. Moreover, changes in $\lambda$ can also produce transitions between dynamic regimes. This is illustrated in Fig. 6, which is based on the phase diagram obtained from our model and depicted in Fig. 5.

The emergence of the oscillatory behavior in a narrow frequency band is shown in Fig. 6 using the solid (blue) region. Oscillatory behavior is a hallmark of learning effects, which are modeled in our NP model using the adaptation (decrease) of excitatory threshold $k_{1}$ from its original value of 3 to 2 , while the inhibitory threshold $k_{2}$ remained unchanged. Note, that in the case of high values of both excitatory and inhibitory thresholds $k_{1}=k_{2}=3$ no oscillatory behavior takes place, rather the dynamics converges to fixed points (not shown). In 


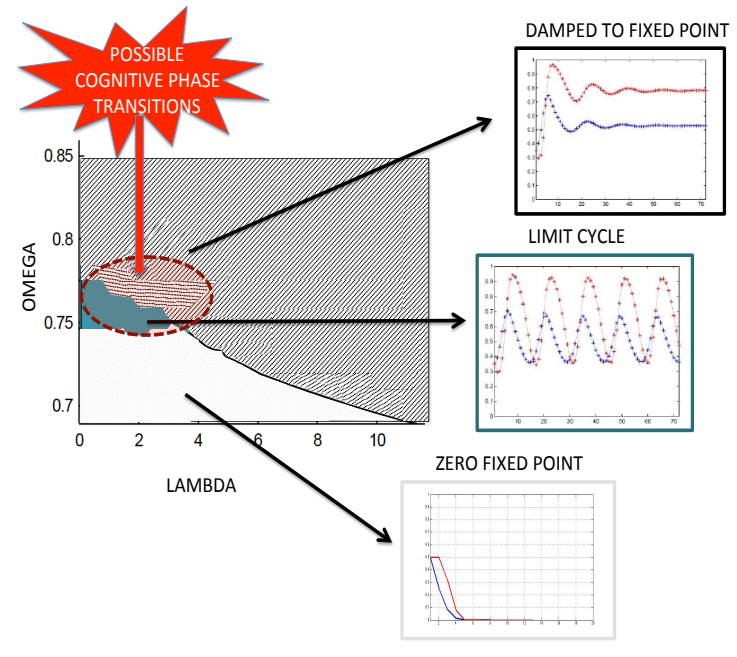

Fig. 5. Dynamic regimes illustrated over the phase diagram in the $\lambda$ versus $\omega$ space. The solid dark region correspond to conditions with limit cycle oscillations created as the result of learning. Transitions from limit cycle regime to fixed point (dashed area) indicate the absence of learnt stimulus. The region corresponding to the hypothetic cognitive phase transitions is circled by dashed line (red).

short, $k_{1}$ may be indeed a control parameter of the learning a category formation process.

\section{CONCLUSIONS}

The main goal of this paper has been introducing a graph theoretical model featuring phase transition behavior, to interpret learning effects observed in experiments. Special emphasis has been on phase transitions in the dynamics of our model which we have be able to control using basic model parameters, such the threshold of a unit to respond to the interactions by its neighbors $\left(k_{1}, k_{2}\right.$, and the extent of the longrange interaction obeying power law behavior $(\lambda)$, as well as the role of inhibitory nodes $(\omega)$. We argued that the structural changes in the network lead to changes in the percolation dynamics, which in turn can be responsible for the sudden behavioral transition of gerbils from a naive to trained state.

In a broader context, we propose a plausible argument for the widely observed "aha" moment of recognition. In the "aha" moment the subject, animal and human alike, suddenly becomes aware of some deeper connections in the world around us or inside us, which are relevant to us. These preliminary results should be analyzed in details to derive conclusive results on the central questions on how we perceive our world.

\section{ACKNOWLEDGMENT}

This work has been conducted with partial support of NSF DMS grant 13-11165 to Robert Kozma, in collaboration with Leibniz Institute of Neurobiology, Magdeburg University, Germany. Special thanks are due to Prof. Frank Ohl, Dr. Andreas Schulz and Dr. Tim Wanger for the gerbil experiments and valuable discussions. Prof. Sanqing Hu's group at Hangzhou Dianzi University has provided us crucial help in data analysis and interpretation.

\section{REFERENCES}

[1] Balister, P., Bollobás, B., and Kozma, R., Large deviations for mean field models of probabilistic cellular automata, Random Structures \& Algorithms 29, (3), 399-415, (2006).

[2] Freeman W.J. 1975. Mass Action in the Nervous System Academic Press

[3] Freeman, W.J. The Physiology of Perception, Scientific American, 264, 78-85, (1991).

[4] Freeman, W.J. Mechanism and significance of global coherence in scalp EEG, Current Opinion in Neurobiology, 31, 199-205, (2015).

[5] Hu, S.Q., Dai, G., Worrell, G., Dai, Q., and Liang, H., Causality analysis of neural connectivity: Critical examination of existing methods and advances of new methods, IEEE Trans Neural Networks, 226: 829844, 2011.

[6] Hu, S.Q., F. Ohl., A. Schulz, T. Wanger, J. Xue, J. Zhang, F. Bao, Q. Dai, Y. Sokolov, R. Kozma Neural Mechanisms of Category Learning in the Auditory Cortex of Gerbils, (in preparation), 2016.

[7] Janson, S., Kozma, R., Ruszinkó, M., and Sokolov, Y., Bootstrap percolation on a random graph coupled with a lattice, arXiv: $1507.07997 \mathrm{v} 2$ [math.CO]

[8] Kozma, R., Puljic, M., Balister, P., Bollobas, B., Freeman, W.J. Phase transitions in the neuropercolation model of neural populations with mixed local and non-local interactions, Biological Cybernetics, 92(6): 367-379 (2005).

[9] Kozma, R., T. Fukuda. Intentional dynamic systems: fundamental concepts and applications, International Journal of Intelligent Systems, 21(9): 875-879 (2006).

[10] Kozma, R., W.J. Freeman, W.J. The KIV model of intentional dynamics and decision making, Neural Networks, 22(3): 277-285 (2009).

[11] Kozma, R., Puljic, M. Learning effects in coupled arrays of cellular neural oscillators, Cognitive Computation, 5(2): 164-169 (2013).

[12] Kozma, R., S.Q. Hu. Stochastic causality, criticality, and non-locality in brain networks: Comment on" Foundational perspectives on causality in large-scale brain networks" by M. Mannino and SL Bressler, Physics of Life Reviews (2015).

[13] Kozma, R., Ruszinko, M., and Sokolov, Y. Percolation on a power-lawlike random graph coupled with a lattice. Part II: The case of two types of nodes, in progress, (2016).

[14] Ohl, F.W., Scheich, H., and Freeman W.J. Change in pattern of ongoing cortical activity with auditory category learning, Nature, 412(6848):7336, (2001).

[15] Ohl, F.W. Commentary by Frank Ohl on the Creation of Meaning in the Brain - Cortical Neurodynamics During Category Learning, In Cognitive Phase Transitions in the Cerebral Cortex-Enhancing the Neuron Doctrine by Modeling Neural Fields, R. Kozma, W.J. Freeman, pp. 147-159. Springer International Publishing, 2016.

[16] Sokolov, Y., R. Kozma, Stability of dynamic brain models in neuropercolation approximation, IEEE International Conference on Systems, Man and Cybernetics (SMC), 2230-2233 (2014). 